\title{
A NOVEL STOCHASTIC MODELLING APPROACH FOR ELECTRIC VEHICLE CHARGING POWER AND ENERGY REQUIREMENTS
}

\author{
Amir Fazeli \\ Substation Automation Solutions, Smart Grid Research Centre \\ Alstom Grid Ltd, \\ Stafford, St Leonards Avenue, ST17 4LX, UK \\ amir.fazeli@alstom.com
}

\author{
C. Mark Johnson, Mark Sumner, Edward Christopher \\ Power Electronics Machines and Control Group \\ University of Nottingham \\ Nottingham, University Park, NG7 2RD, UK \\ mark.johnson@nottingham.ac.uk
}

\begin{abstract}
Electrification of heat and transport in addition to integration of intermittent renewable resources into the existing electricity network is expected to occur in near future. Such a transformation is expected to force the operation of the electricity power system at different levels to its limits. A novel bottom up modelling approach for quantifying temporal variation of electric vehicle charging power and energy is presented in this paper which can be used to accurately investigate the effect of charging different penetration levels of electric vehicles within the low voltage distribution network. This EV charging model is further used for development of a deterministic control algorithm for regulating power flows at the low voltage level.
\end{abstract}

Index Terms-Electric vehicle, stochastic load modelling, electric vehicle battery, distributed energy resources

\section{INTRODUCTION}

$\mathrm{T}$ he increasing uptake of electric vehicles is expected to have a number of adverse effects on the radial low voltage distribution network including [1]:

- Increased voltage drop at the consumer end of LV feeders

- Overloading of distribution transformers and feeders

- Increased network losses

- Voltage unbalance

- System frequency deviations

- Current harmonics and reduced power quality

The impacts of different penetrations of Electric Vehicle (EV) charging on distribution transformers, feeder loading, voltage and power losses are investigated in [2]. Simulation results presented in [2] demonstrate that the distribution transformer and underground cables serving customers will be significantly overloaded. In addition to that a high penetration of EVs would cause the voltage at the end of the feeders to drop below 0.94p.u and increase power loss within the system. It is worth noting that quantifying the temporal variation of EV load through the relevant modelling technique is an essential element for studying the effect of integrating EVs on the distribution network operation. In addition to that, provided that there is a certain degree of flexibility in the charging of each unit, this modelling platform would allow for individual EVs to participate in the operation of any active management algorithm for regulating power flows at the low voltage level. This would in turn significantly reduce the negative effects that would otherwise occur if EVs were allowed to charge freely. A novel bottom-up modelling approach for quantifying the temporal variation of $\mathrm{EV}$ availability for charging and it's charging energy requirement throughout a day is presented in this paper. The presented EV charging model has been utilised for both development and testing of the Community Power Flow Control algorithm (CPFC) a novel deterministic control algorithm for regulating power flow within a section of the distribution networks. The structure and the logic behind the CPFC algorithm will be presented in a future publication. An overview of some of the available modelling techniques for quantifying the temporal variation of $\mathrm{EV}$ charging requirements has been provided in section II. The proposed novel modelling technique for EV charging in addition to the simulation result obtained from this model, are presented in section III and IV of this paper respectively.

\section{LITERATURE REVIEW}

An EV charging model has been presented in [2].This model essentially uses two probability distribution functions to randomly quantify the "charging start time" and the "State of Charge". However these distributions do not appear to be based on any transport data. In addition, this model only quantifies charging for the last trip of the day, so visits to the house charging point at interim times are not included. A similar technique for temporal quantification of EV charging has been presented in [3]. This technique uses the National Household Transport Survey (NHTS) data set [4], to obtain a distribution of "last journey ending time" and "daily driving distance" which are then used to randomly quantify the vehicle arrival time at the dwelling and its total energy 
consumption for the day. Similar to [2], this model does not attempt to quantify the temporal variation of vehicle availability at the dwelling throughout the day. Excluding the possibility of charging throughout the day, as presented in [2], [3] is expected to result in overestimation of the charging power requirements for the late evening hours. An agent based EV model has been presented in [5] which uses random distributions to determine individual departure times as well as type and detailed location of the next activity in the city. For every individual agent, the model generates the total distances driven and energy consumed, however no detail about the statistical method used for the computation of these variables has been provided. As explained above the majority of the published modelling approaches are unable to quantify the availability of EVs for charging and their charging energy requirements. The modelling approach presented in this paper, on the other hand, relies on the application of appropriate statistical techniques to information extracted from a comprehensive and detailed transport data set. This ensures that the proposed modelling approach is a reliable and an accurate method for stochastic and realistic quantification of vehicle availability for charging and journey energy consumption.

\section{THE ELECTRIC VEHICLE CHARGING MODEL}

The developed EV charging model comprises two elements. The first element is a novel stochastic method for temporal quantification of vehicle availability for charging and its journey energy consumption. The second element is a lithium ion battery model based on an RC equivalent circuit, whose state-of-charge is linked to the journey energy consumption. The structure behind the two elements of the EV charging model in addition to the simulation results obtained from this model are included in the next section.

\section{A. Vehicle availability for charging and journey energy consumption model}

A vehicle's position and commutation throughout a day are assumed to be described by stochastic temporal variables with a changing likelihood across the day. This is represented by a Markov Chain [6] with a discrete state space to define the location and hence availability for charging. The state transition probability values are extracted from the NHTS data set [4], which contains specific information about individual journeys that 240,000 participants had taken in a day with a one minute resolution. A Monte Carlo technique [7] is then applied to the resultant Markov Chain to obtain the state transition for every individual vehicle throughout the day. The energy consumption for each vehicle trip generated by the Monte-Carlo model is estimated by using the NHTS data to generate a journey distance from the journey duration. The resultant value of journey distance is then multiplied by the specific energy consumption rate of the vehicle to quantify the energy consumption during the journey. In this model it is assumed that charging the vehicle's battery only takes place when the EV is parked at the dwelling.

\section{1) Construction of the Markov Chain}

In order to quantify the variable likelihood of journeys taken throughout a day and the availability of the vehicle at the dwelling, the following three states of vehicle mobility have been defined:

- State One - vehicle parked at the dwelling

- State Two - vehicle on a journey

- State Three - vehicle parked at a remote location other than the dwelling

Using every participant's journey diary which contains information about every journey's start and end time, the participant's location and journey distance, it is possible to create a daily vehicle mobility profile for every participant. This profile indicates how transition occurs between the three vehicle mobility states throughout a day. Fig.1 shows an example of a vehicle mobility state profile created from the diaries of four participants. It is evident that all four participants leave their dwellings during the interval 7:25 8:25 hours. They mostly park their vehicle at a remote location during mid-day hours (with the exception of a few sporadic journeys) and make a return journey back to the dwelling during the interval 15:30 - 17:00 hours. Vehicle four then takes an additional journey in the evening hours, however the remaining vehicles are parked at the dwelling and hence available for charging during the evening hours.

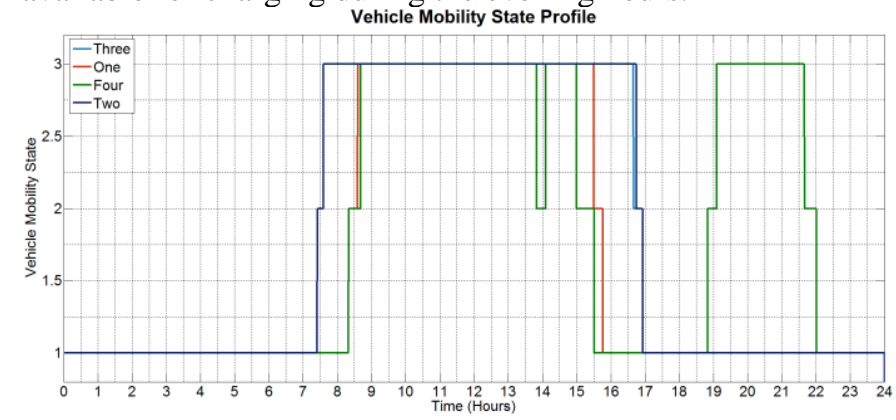

Fig.1.Vehicle mobility state profiles obtain from NHTS

By filtering all the journey diaries obtained from the data set, the journey patterns of all participants are obtained in the three state vehicle mobility format. Using the filtered data, the total number of transitions between every one of the states and its two counterpart states is determined, for every minute of a day. Having quantified these state transition values for every minute of a day, the probability of transition between different states is simply calculated using (1).

$$
P_{t_{m n}}=\frac{X_{t_{m n}}}{Y_{t_{m}}}
$$

Where $X_{t_{m n}}$ is the total number of transitions from state $\mathrm{m}$ to state $\mathrm{n}$ during the one minute interval at time $\mathrm{t}$ and $Y_{t_{m}}$ is the total number of vehicles in state $\mathrm{m}$ at time $\mathrm{t}$. Since three vehicle mobility states have been defined in this study, there are nine combinations of state transition values for every minute, only one of which can occur during any particular one minute interval. This is illustrated in Fig.2, which shows the three mobility states and the nine possible state transitions at time t. Since any transition between state 1 and state 3 has to take place via state 2 , the probabilities of a direct transition between state 1 and state 3 are effectively zero. The obtained state transition probability values can be placed in individual $3 \times 3$ sub-matrices, one for every minute of the day. 


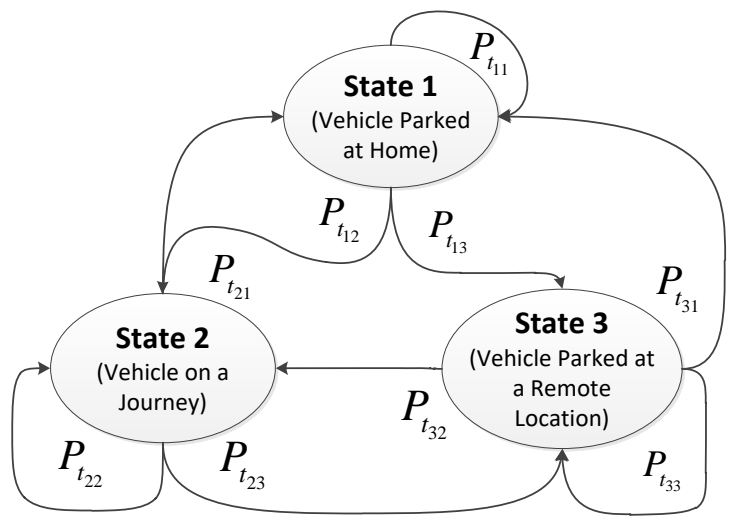

Fig.2.Three mobility states and the different state transition possibilities at time $\mathrm{t}$

Fig.3 shows the state transition probability sub-matrix at minute t.

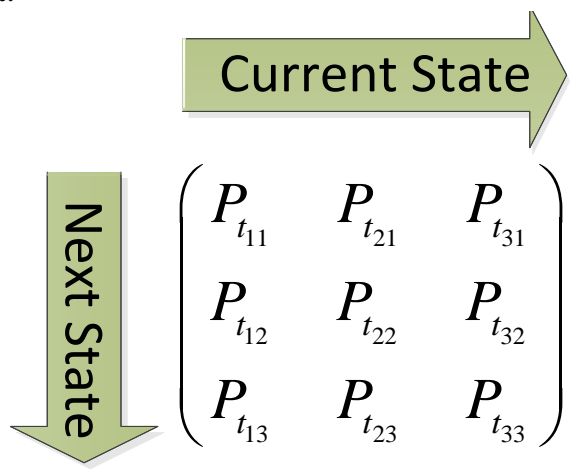

Fig.3. State transition probability sub-matrix at minute $t$

The columns of this sub-matrix represent the possible present states and the rows represent the possible states at the next time step. Every matrix element represents the probability of transition from the present state to the next state. For example $P_{t_{12}}$ represents the probability of transition to state 2 (vehicle starts a journey) if the current state is state 1 (vehicle parked at the dwelling). Likewise $P_{t_{22}}$ represents the probability of remaining in state 2 at time t. This forms the basis of a classical Markov Chain with a finite discrete state space, in which state transition is dependent on the previous state together with the probability of the state changing. Repeating the aforementioned procedure for every minute of a day, 1440 unique sub-matrixes are created (one for each minute of the day), which are then grouped together to form the resultant State Transition Probability Matrix (STPM). It is worth noting that journey patterns are expected to be different between weekdays and weekend days. To accommodate the different behaviour patterns between weekday and weekend, the original survey data set is divided into two groups and two separate state transition probability matrices are created. This distinction ensures that the variation of journey probability between weekdays and weekend days are incorporated in this model.

2) Application of the Markov chain Monte Carlo technique

The Markov-Chain Monte Carlo technique is a convenient way to model the evolution of vehicle states throughout the day while respecting the underlying behavioural statistics of the entire vehicle population. Since the probability of occurrence of state 1 at time 00:00 hour is much higher than the probability of occurrence of any other states, it is assumed that every vehicle is in state 1 at the start of the simulation. The ensuing state transition probabilities at each subsequent step can be determined by repeatedly comparing a random number with the cumulative elements of the state transition probability vector obtained from (3) [7]. The value of the random number will then determine the state at the next time step. A state transition only occurs if the cumulative probability value is greater than the random number, otherwise the vehicle stays in its current state during that time step.

$$
\left.\begin{array}{c}
\mathbf{q}_{t+1}=\mathbf{P}_{t} \mathbf{s}_{t} \\
q_{1} \\
q_{3}
\end{array}\right]_{t+1}=\left[\begin{array}{ccc}
P_{11} & P_{21} & P_{31} \\
P_{12} & P_{22} & P_{32} \\
P_{13} & P_{23} & P_{33}
\end{array}\right]_{t} \cdot\left[\begin{array}{c}
s_{1} \\
s_{2} \\
s_{3}
\end{array}\right]_{t}
$$

Here $\mathbf{P}_{t}$ is the state transition probability matrix at time $\mathbf{t}, \mathbf{S}_{t}$ is the state vector containing binary values which represent the active state at the current time step and $\mathbf{q}_{t+1}$ is the state transition probability vector at the next time step. Note that the sum of state transition probabilities from any one state to any subsequent state must be unity as shown in (4).

$$
\sum_{i=1}^{3} q_{i}=1
$$

Performing this technique at every minute throughout the day using the appropriate elements of the state transition probability matrix, results in the synthetic generation of the vehicle mobility state profile. Fig.4 shows three examples of the resultant vehicle mobility state profiles over a week day.

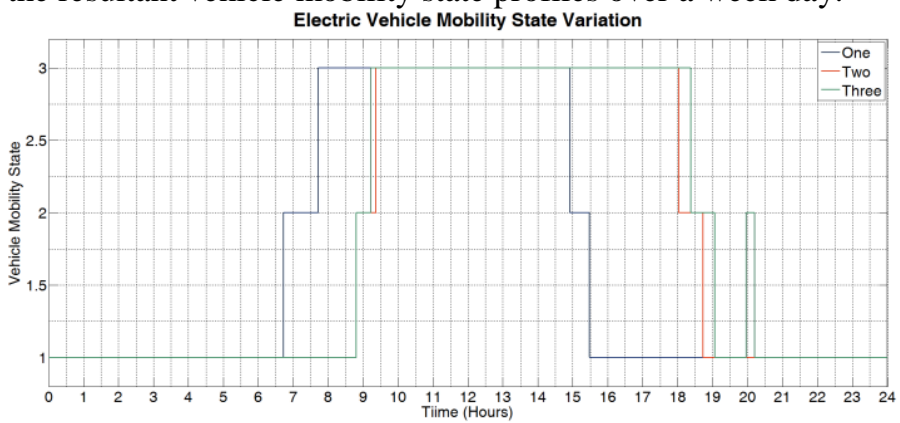

Fig.4.Three individual vehicle mobility state profile

For example it is evident that the third EV takes three journeys during the day starting at $08: 45$, of 35 minutes, 40 minutes and 15 minutes duration respectively. Most of the journey patterns have similar characteristics, with a greater likelihood of journeys (i.e. state 2) during the morning and evening hours, parking at the dwelling (i.e. state 1) overnight and during the early morning hours, and parking at a remote location (i.e. state 3 ) around midday and sometimes during the late evening hours. These characteristics reflect the behaviour of the population which was used to generate the filtered survey data. The duration of each journey is represented by the intervals of time spent in state 2, while the vehicle's 
availability at the dwelling for charging is represented by the intervals of time spent in state 1 . Since there is a direct correlation between journey distance and journey duration it is reasonable to use this correlation in combination with the value of journey duration obtained from the mobility state profile to estimate the journey distance. In order to generate the correlation statistic, the NHTS data is firstly filtered into 26 journey duration bins each with a 10 minute width, followed by further filtration of every duration bin into 300 distance bins, each with a width of 1 mile. Finally, the cumulative probability distribution of journey distance for each one of the journey duration bins is obtained from the resultant filtered distributions. Having obtained the journey duration value from the vehicle mobility state profile, the respective cumulative probability distribution is selected. By comparing a normally distributed random number (between 0 and 1) with the selected cumulative probability distribution, an estimated journey distance value can be generated, that correctly reflects the statistical variation in the underlying survey data. Having quantified every journey's distance, each journey's energy consumption is simply calculated using (5).

$$
E_{J}=D_{J} \times E C R
$$

$E_{J}$ is journey energy consumption in $\mathrm{kWh}, D_{J}$ is journey distance in miles and ECR is the journey energy consumption rate, which in this study is assumed to be constant at $0.34 \mathrm{KhW} / \mathrm{mile}[8]$. For example the application of these steps on the mobility profile for vehicle three (shown in Fig.4) entails $3.7 \mathrm{kWh}, 4.8 \mathrm{kWh}$ and $1.2 \mathrm{kWh}$ of consumed energy during the three journeys.

\section{B. Lithium ion battery and charger model}

A simplistic lithium ion battery model has been presented in [9]. This model is essentially based on the operation of individual battery cells represented through an equivalent RC circuit. As the modelling steps presented in [9] were transparent and battery parameters were also available from the literature [10], a similar modelling technique has been adopted in this project, to represent a lithium ion battery. The equivalent RC circuit shown in Fig.5 is used to represent a single 6Ah lithium ion battery cell.

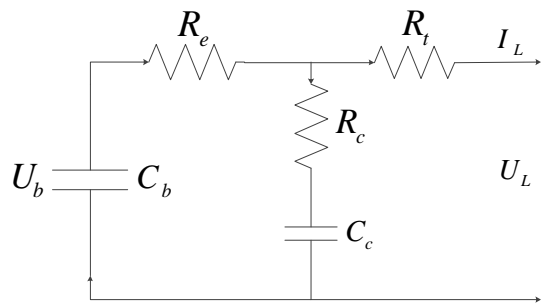

Fig.5.RC Equivalent circuit of a lithium ion battery cell

This circuit consists of two capacitors $\left(C_{b}, C_{c}\right)$ and three resistors $\left(R_{t}, R_{e}, R_{c}\right)$. The capacitor $C_{c}$, which has a small capacitance, mostly represents the surface effects of a battery. A large capacitor $C_{b}$ represents bulk charge storage within the battery. Resistors $R_{t}, \boldsymbol{R}_{e}$ and $\boldsymbol{R}_{c}$ are called the terminal resistor, the end resistor and the capacitor resistor respectively.
$U_{b}$ and $U_{c}$ are the voltages across $C_{b}$ and $C_{c}$ respectively. The electrical behaviour of the circuit can be expressed by (6) and (7).

$$
\begin{aligned}
& {\left[\begin{array}{l}
\frac{d U_{b}}{d t} \\
\frac{d U_{c}}{d t}
\end{array}\right]=\left[\begin{array}{ll}
\frac{-1}{C_{b}\left(R_{e}+R_{c}\right)} & \frac{1}{C_{b}\left(R_{e}+R_{c}\right)} \\
\frac{1}{C_{c}\left(R_{e}+R_{c}\right)} & \frac{-1}{C_{c}\left(R_{e}+R_{c}\right)}
\end{array}\right]\left[\begin{array}{l}
U_{b} \\
U_{c}
\end{array}\right]+\left[\begin{array}{c}
\frac{-R_{c}}{C_{b}\left(R_{e}+R_{c}\right)} \\
\frac{-R_{e}}{C_{c}\left(R_{e}+R_{c}\right)}
\end{array}\right]\left[I_{L}\right]} \\
& {\left[U_{L}\right]=\left[\begin{array}{ll}
\frac{R_{C}}{\left(R_{e}+R_{c}\right)} & \frac{R_{e}}{\left(R_{e}+R_{c}\right)}
\end{array}\right]\left[\begin{array}{l}
U_{b} \\
U_{c}
\end{array}\right]+\left[-R_{t}-\frac{R_{e} R_{c}}{\left(R_{e}+R_{c}\right)}\right]\left[I_{L}\right]}
\end{aligned}
$$

$U_{L}$ and $I_{L}$ in (6) and (7) represent the terminal voltage and current from the battery cell. Using this battery cell model, a number of cells are connected in series to make up the desired battery pack capacity. The charging characteristics and specification of the Nissan Leaf battery has been obtained from [8], and is used in this model. Therefore a $24 \mathrm{kWh}$ battery pack with a constant charging power of $6.6 \mathrm{~kW}$ has been modelled to represent the EV battery. In order to regulate the battery's charge/discharging power in addition to its state of charge, a model for a battery charge controller is required. The model presented in [11] has been employed for this purpose. Having assumed that the charging power $P_{\text {charging }}$ is constant at $6.6 \mathrm{~kW}$, the charging current is simply calculated according to (9). This assumes a constant efficiency of $98 \%$ for the charger $\left(\varepsilon_{C}\right)$, and $90 \%$ for the power electronic rectifier $\left(\varepsilon_{P E}\right)$ [11].

$$
I_{L}=\frac{P_{\text {charging }} \times E V_{A}}{V_{B} \times \varepsilon_{C} \times \varepsilon_{P E}}
$$

$V_{B}$ represent the voltage across the battery pack which is calculated by multiplying the number of battery cells with $U_{L}$ obtained from (7). $E V_{A}$ is a binary variable representing the availability of the $\mathrm{EV}$ at the dwelling (i.e. 1 or 0 depending on state). The variation of State of Charge (SOC) is simply quantified by integrating the value of the battery current $I_{L}$ over time. The battery charger ensures that the EV battery isn't discharged below $15 \%$, and charged above $80 \%$ of its maximum SOC. The state of charge of an EV's battery is updated at the end of every journey with a new value according to (10).

$$
S O C_{t}=S O C_{t-x}-S O C_{j}
$$

$S O C_{t-x}$ represents the state of charge at the start of the journey and $S O C_{j}$ is the reduction in state of charge due to the journey. Upon entering state 1 the EV starts to charge until either the mobility state changes (i.e. EV becomes unavailable) or the EV's battery becomes fully charged. This assumes that the EV owner plugs in the EV for charging upon arrival at home.

\section{Simulation RESUlts}

After integrating the vehicle mobility model with the battery model, the temporal variation of the SOC and charging power can be obtained. Fig. 6 shows the variation of the SOC for the 
three EVs shown in Fig.4. The SOC variation shows the following three distinctive periods:

- Increasing SOC as the EV is being charged at the dwelling

- Constant SOC (i.e. less than $80 \%$ ) as the EV is parked at a remote location and not charging

- Reducing SOC as the EV is on a journey consuming energy

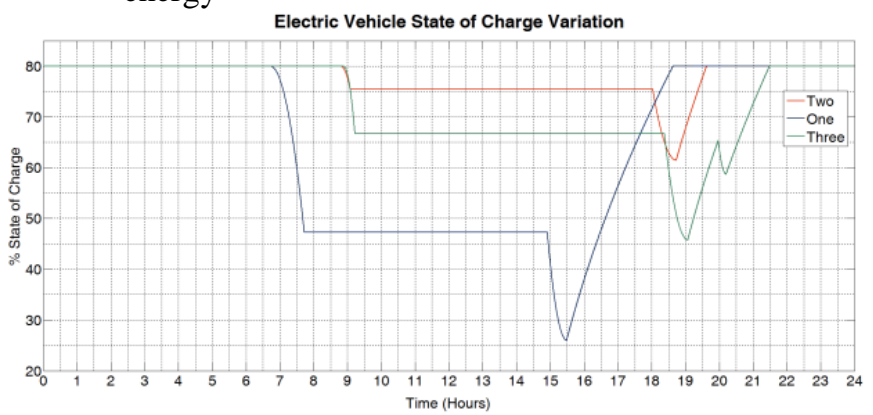

Fig.6.SOC variation of three EVs obtained from simulation

Having quantified the variation of SOC and EV charging power, it is possible to include every EV as a shed-able load in the CPFC's operation. However since prolonged EV shedding is unrealistic and undesirable to the EV user, different shedding bands have been created and randomly assigned to different users at the start of every simulation run. It is envisaged that every EV user will select the desirable maximum and minimum shedding times via a smart meter interface.

\section{CONCLUSION}

A novel probabilistic modelling approach for quantifying the temporal variation of the power and the energy required for charging individual $\mathrm{EV}$ units is developed and presented in this paper. This model is based on application of a Monte Carlo simulation on data extracted from a transport survey. Integration of this probabilistic technique with a lithium ion battery model permitted the realisation of a stochastic model for EV energy requirements. In order to prove the validity of the proposed modelling technique for the generation of synthetic vehicle mobility patterns, the variation of every state probability with time is obtained from the NHTS survey and compared with aggregated Monte-Carlo simulation runs. The preliminary findings obtained from that investigation shows close correlation between the simulation results and will be presented in more depth in a forthcoming publication. This EV charging model is then used for development of CPFC, a novel deterministic control algorithm developed by the authors, for regulating power flows within different sections of the distribution network. CPFC's structure and logical operation in addition to a comprehensive smart grid control framework will be presented in a future publication.

The presented EV charging model at its current stage merely represents every EV as a load since the V2G operation has been omitted. Inclusion of V2G is considered an improvement to the presented model's functionality and has been assigned as future work. That work will involve devising additional conditions for EV charger in order to distinguish between charging and $\mathrm{V} 2 \mathrm{G}$ modes of operation.

\section{REFERENCES}

[1] P. Papadopoulos, L. M. Cipcigan, N. Jenkins, and I. Grau, "Distribution networks with Electric Vehicles," in Universities Power Engineering Conference (UPEC), 2009 Proceedings of the 44th International, 2009, pp. 1-5.

P. Papadopoulos, S. Skarvelis-Kazakos, I. Grau, B. Awad, L. M. Cipcigan, and N. Jenkins, "Impact of residential charging of electric vehicles on distribution networks, a probabilistic approach," in Universities Power Engineering Conference (UPEC), 2010 45th International, 2010, pp. 1-5.

[3] L. Kelly, A. Rowe, and P. Wild, "Analyzing the impacts of plug-in electric vehicles on distribution networks in British Columbia," in Electrical Power \& Energy Conference (EPEC), 2009 IEEE, 2009, pp. 1-6.

[4] U. S. D. o. Transportation, "The National Household Travel Survey (NHTS)," N. D. Center, Ed., ed. NHTS Data Center, 2009.

[5] S. Acha, K. H. van Dam, and N. Shah, "Modelling spatial and temporal agent travel patterns for optimal charging of electric vehicles in low carbon networks," in Power and Energy Society General Meeting, 2012 IEEE, 2012, pp. 1-8.

[6] S. R. Walter R. Gilks, David J. Spiegelhalter, Markov Chain Monte Carlo in practice: Chapman and Hall/CRC (1 Dec 1995), 1996.

[7] H. F. L. Dani Gamerman, "Markov chain Monte Carlo: stochastic simulation for Bayesian inference," ed: Chapman and Hall/CRC; 2 edition (10 May 2006), 2006, p. 344 pages.

[8] NISSAN. (2012, 11/07/2013). NISSAN LEAF. Available: http://www.nissanusa.com/electric-cars/leaf/versions-specs/

[9] R. X. a. J. F. Hongwen He *, "Evaluation of Lithium-Ion Battery Equivalent Circuit Models for State of Charge Estimation by an Experimental Approach," Energies, vol. 4, 2011.

[10] A. A. P. Valerie H. Johnson, "Temperature-Dependent Battery Models for High-Power Lithium-Ion Batteries," 2000.

[11] J. F. D.P. Jenkins *, D. Kane, "Model for evaluating impact of battery storage on microgeneration systems in dwellings," Energy Conversion and Management, vol. 49, 2008.

\section{BIOGRAPHIES}

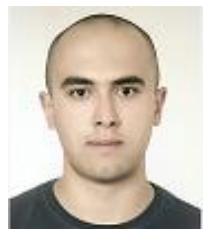

Amir Fazeli received the BEng honors degree in Electrical and Electronic Engineering from the University of Liverpool in 2008, and the MSc in Power Electronics and Drives from the University of Nottingham in 2009. In March 2013 he completed his PhD studies of Electrical and Electronic Engineering at the University of Nottingham, where he focused on development of novel control techniques for regulating power flow within microgrids. In November 2013 Dr. Fazeli joined Alstom Grid Ltd where he is currently a research technologist.

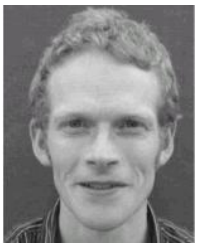

C. Mark Johnson received the B.A. degree in Engineering and the $\mathrm{PhD}$ degree in Electrical Engineering from the University of Cambridge, in 1986 and 1991, respectively. In 2006, he was granted a Personal Chair at the University of Nottingham, where he is involved in power semiconductor devices, power device packaging, reliability, thermal management, power module technologies, and power electronic applications. Prof Johnson is currently leading the EPSRC National Centre of Excellence for Power Electronics.

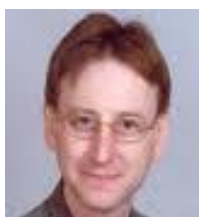

Nottingham.

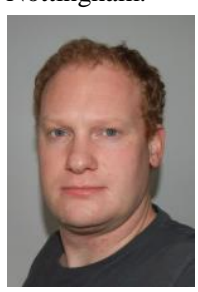

Mark Sumner received his BEng degree in Electrical and Electronic Engineering from Leeds University in 1986. Prof. Sumner received his PhD in 1990, and after working at Nottingham as a Research Assistant, was appointed Lecturer in October 1992 and Senior Lecturer in 2004. In 2011 he was appointment a Professor of Electrical Energy Systems at the University of

Edward Christopher obtained his B.Eng degree and $\mathrm{PhD}$ in Electrical and Electronic Engineering from The University of Nottingham in 2003 and 2008 respectively. In 2007 Dr. Christopher was employed as a Research Fellow in the Power Electronics, Machines and Control Group where he worked on power electronics systems for automotive, marine and renewable energy applications. In 2012 he became Lecturer of Renewable Energy Integration and Power Electronics. 